\title{
Semantic priming in the pronunciation task: The role of prospective prime-generated expectancies
}

\author{
DENNIS E. KEEFE \\ Purdue University, West Lafayette, Indiana \\ and \\ JAMES H. NEELY \\ State University of New York, Albany, New York
}

\begin{abstract}
In the relatedness proportion effect, semantic priming increases with an increase in the probability that a word prime will be followed by a semantically related word target. This effect has frequently been obtained in the lexical decision task but not in a pronunciation task. In the present experiment, relatedness proportion was manipulated in two pronunciation tasks, one with and one without nonword targets, using category names as primes. In both tasks, a relatedness proportion effect occurred for high-dominance category exemplars but not for low-dominance category exemplars. These results converge with recent lexical decision results in suggesting that semantic priming in pronunciation is affected by a prospective prime-generated expectancy that is modulated by the relatedness proportion.
\end{abstract}

In the semantic priming effect, responses are faster and/or more accurate when a target word is preceded or accompanied by a semantically related prime rather than an unrelated prime (e.g., see Meyer \& Schvaneveldt, 1971; Neely, 1976; see also Neely, in press, for a review). In the lexical decision task (LDT), in which subjects make word/nonword decisions about the target, semantic priming effects increase in size as the proportion of related word-prime/word-target trials (the relatedness proportion) increases (e.g., see de Groot, 1984; den Heyer, 1985; den Heyer, Briand, \& Dannenbring, 1983; Neely, Keefe, \& Ross, 1989; Seidenberg, Waters, Sanders, \& Langer, 1984; Tweedy, Lapinski, \& Schvaneveldt, 1977). In the present research, we test Neely and Keefe's (1989) and Neely et al.'s (1989) theoretical analysis of the two mechanisms responsible for this relatedness proportion effect (hereafter RPE) in the LDT.

Portions of this research were presented at the 1984 and 1988 meetings of the Midwestern Psychological Society in Chicago and were conducted by Dennis E. Keefe in partial fulfillment of the requirements for the MS degree at Purdue University, under the direction of James $H$. Neely. We thank Henry L. Roediger III and Richard Schweickert, who also served on the MS committee. We also thank Gordon Logan and David Balota for their helpful comments. Finally, we thank Kim Brady and Kent Ross for testing some of the subjects. This research was supported by an XL grant from Purdue Research Foundation and by NICHHD Grant R01 HD15054. Requests for reprints should be sent to James H. Neely, Department of Psychology, State University of New York at Albany, Albany, NY 12222.
The Roles of Expectancy and Semantic Matching in the RPE in the LDT

According to Neely and Keefe (1989), one mechanism that produces the RPE is an expectancy strategy (e.g., see den Heyer et al., 1983; Neely, 1977; Posner \& Snyder, 1975). When employing this strategy, subjects use the prime to generate an expectancy for a set of semantically related targets that would likely follow that prime (cf. Becker, 1980). If the target is a member of this expectancy set, lexical access for that target is facilitated, relative to a neutral priming condition (e.g., a string of $\mathrm{Xs}$ ), in which no expectancy set is generated; if the target is not a member of this expectancy set, lexical access for that target is inhibited, relative to the neutral priming condition. Because the expectancy mechanism is assumed to affect the speed of lexical access for the target, it is called a prelexical priming mechanism. The RPE is explained by assuming that subjects become more likely to generate an expectancy set of items related to the prime as the relatedness proportion increases, thereby increasing priming.

In Neely and Keefe's (1989) analysis, the second mechanism responsible for the standard RPE in the LDT is a semantic matching mechanism (after Neely, 1977). This mechanism is a specific instantiation of what others have referred to as postlexical priming mechanisms (e.g., Balota \& Lorch, 1986; de Groot, 1984, 1985; de Groot, Thomassen, \& Hudson, 1982; den Heyer, 1985; Forster, 1979, 1981; Norris, 1986; Ratcliff \& McKoon, 1988; Seidenberg et al., 1984; Stanovich \& West, 1983). According to Neely and Keefe (1989), after the prime's and 
target's lexical and semantic representations have been activated, but before the subjects have had time to complete their lexical decision and/or response to the target, the subjects may check whether the target is semantically related or unrelated to the prime that preceded it. If it is related, a "word" decision/response bias ensues, thereby facilitating responses to related word targets; if it is unrelated, a "nonword" decision/response bias ensues. (Why these word and nonword biases occur will be explained shortly.) Because a "nonword" bias takes time to overcome, reaction times (RTs) to unrelated word targets should be slowed relative to a neutral priming condition, in which this "nonword" bias is not present (because subjects do not check for target-prime relatedness for neutral primes). Thus, as does the prelexical expectancy mechanism, the postlexical semantic matching mechanism produces both facilitation from related primes and inhibition from unrelated primes in the LDT. However, because this facilitation and inhibition are produced by decision/response processes that occur after lexical access for the target has occurred, semantic matching is said to be a postlexical priming mechanism.

The semantic matching mechanism can also account for the RPE. (See also de Groot, 1984, 1985, den Heyer, 1985, and Seidenberg et al., 1984, for analyses of how their postlexical priming mechanisms can account for the RPE.) However, according to Neely et al. (1989), the semantic matching mechanism is not influenced by relatedness proportion per se but rather is being influenced by a probability that had previously been perfectly confounded with the relatedness proportion. This probability (called the nonword ratio) is the probability that a target is a nonword, given that it is unrelated to the word prime that precedes it.

To understand the confounding of the nonword ratio and the relatedness proportion, consider two groups tested by Neely et al. (1989, Experiment 1$)$ in their LD'T. In these two groups, category names (e.g., BIRD) served as primes and category exemplars as targets. In all priming conditions with word targets, high- and low-dominance exemplars (e.g., robin and swan, respectively) served equally often as targets. For one group of subjects, for every 96 trials, there were 42 related word-prime/wordtarget trials, 6 unrelated word-prime/word-target trials, and 48 unrelated word-prime/nonword-target trials. (Nonword targets may be considered as unrelated to their category-name primes, because they never looked like exemplars of the category-name prime that preceded them.) Because 42 of the 48 word-prime/word-target trials were related, the relatedness proportion was .88 , and because 48 of the 54 trials in which the prime and target were unrelated contained nonword targets, the nonword ratio was .89. Thus, this group was called the "RP $(.88) / \mathrm{NR}(.89)$ group, ' with RP standing for relatedness proportion and NR for nonword ratio. In the RP(.33)/NR(.60) group, for every 96 trials, there were 16 related word-prime/wordtarget trials, 32 unrelated word-prime/word-target trials, and 48 unrelated word-prime/nonword-target trials. Thus, as relatedness proportion increased from .33 to .88 , the nonword ratio increased from .60 to .89 . (Indeed, as long as the overall ratio of word:nonword targets is held constant across the various relatedness proportions and the nonword targets never look like words related to the primes that precede them, a correlation very near +1.0 necessarily exists between relatedness proportion and the nonword ratio.)

As relatedness proportion increased from .33 to .89 in these two groups, overall priming (computed by subtracting RTs in the related priming condition from RTs in the unrelated priming condition) increased by $30 \mathrm{msec}$ (from 37 to $67 \mathrm{msec}$ ) for high-dominance targets and by $29 \mathrm{msec}$ (from 23 to $52 \mathrm{msec}$ ) for low-dominance targets. However, rather than having been produced by increases in the relatedness proportion, these increases in overall priming could have been produced in whole or in part by the confounded increase in the nonword ratio from .60 to .89 . In Neely et al.'s (1989) theoretical analysis, this could have occurred because increases in the nonword ratio would make it more likely that subjects would invoke the semantic matching strategy. This is so because the structure of the LDT is such that subjects can use information concerning target-prime relatedness or unrelatedness to facilitate their binary word/nonword decisions. That is, if the target is related to its prime, the correct response is always "word" (regardless of the nonword ratio). However, if the target is unrelated to its prime, the correct response is more likely to be "nonword," the higher the nonword ratio is. More concretely, in Neely et al.'s $\mathrm{RP}(.88) / \mathrm{NR}(.89)$ group, when the target was unrelated to its prime, the odds were $48: 6$ that the target was a nonword; in the RP(.33)/NR(.60) group, however, when the target was unrelated to its prime, these odds dropped to $48: 32$. Thus, relative to the subjects in the $\operatorname{RP}(.33)$ / $\mathrm{NR}(.60)$ group, the subjects in the $\mathrm{RP}(.88) / \mathrm{NR}(.89)$ group should have been more likely to use the semantic matching strategy because of its greater utility in predicting and facilitating their "nonword" responses. Their greater use of the semantic matching strategy would produce larger priming effects for them.

To examine the degrees to which the increases in relatedness proportion and the nonword ratio were influencing the increases in overall priming, Neely et al. (1989) independently varied the relatedness proportion from .20 to .89 , and the nonword ratio from .08 to .91 , across 12 groups of subjects. (This was accomplished by orthogonally varying the relatedness proportion and the ratio of word:nonword targets; see Neely et al., 1989, for details.) Multiple linear regression analyses that partialed out the separate effects that relatedness proportion and the nonword ratio had on priming showed that increases in both the relatedness proportion and the nonword ratio produced significant isolable increases in priming for highdominance exemplars, whereas only the isolable increases in the nonword ratio significantly increased priming for low-dominance exemplars. More specifically, overall priming for high-dominance exemplars increased by 
$4.1 \mathrm{msec}$ for every . 10 change in relatedness proportion and by $2.7 \mathrm{msec}$ for every . 10 change in the nonword ratio, whereas overall priming for low-dominance exemplars increased by $3.8 \mathrm{msec}$ for every .10 change in the nonword ratio.

On the basis of these results, Neely et al. (1989) concluded that in the standard RPE, in which relatedness proportion and the nonword ratio are confounded, the increase in priming for high-dominance exemplars is produced by a greater utilization of both the expectancy mechanism, which is modulated by relatedness proportion, and the semantic matching mechanism, which is modulated by the nonword ratio. On the other hand, the increase in priming for low-dominance exemplars is produced only by the greater utilization of the semantic matching mechanism, due to the confounded increases in the nonword ratio. Neely et al. (1989) argued that increases in relatedness proportion, which modulate the use of the expectancy mechanism, did not affect overall priming for low-dominance exemplars, because by definition low-dominance exemplars would not be likely to be in the expectancy set generated from the prime. Finally, because the semantic matching mechanism produces a "nonword" bias when the target is found to be unrelated to the prime, for every .10 that the nonword ratio increased there was also a small, but significant, $1.2-\mathrm{msec}$ increase in nonword facilitation-that is, in faster RTs to nonword targets when they follow word primes rather than neutral, XXX primes (e.g., see de Groot, 1984; den Heyer, 1985; Neely, 1976, 1977).

The dissociative effects that relatedness proportion and the nonword ratio had on priming for high- and lowdominance exemplars strongly implies the operation of two separate priming mechanisms. However, it does not necessarily follow that one is a prelexical expectancy mechanism and the other a postlexical semantic matching mechanism, as Neely et al. (1989) claimed. For example, one could argue that rather than modulating the prelexical expectancy mechanism, relatedness proportion influences a postlexical priming mechanism (cf. de Groot, 1984, and Seidenberg et al., 1984) that affects priming only for high-dominance exemplars (e.g., see de Groot, 1985, pp. 294-295) and is different from the postlexical mechanism that is sensitive to changes in the nonword ratio and produces priming for low-dominance exemplars.

To test Neely et al.'s (1989) claim that the prelexical expectancy mechanism contributes to the relatedness proportion effect for high-dominance exemplars but not for low-dominance exemplars in the LDT, one could examine RPEs in a task in which postlexical priming mechanisms do not operate. If an RPE were still found for highdominance exemplars but not for low-dominance exemplars in such a task, the data would favor Neely et al.'s (1989) assertion that a prelexical expectancy mechanism contributes to the RPE in the LDT. If, on the other hand, RPEs were not found in such a task, the data would favor the claim that the RPE is mediated solely by postlexical mechanisms and not by the prelexical expectancy mecha- nism. In the present research, a pronunciation task rather than an LDT is used to invoke this logic. We now turn to our rationale for selecting the pronunciation task.

\section{Does Semantic Matching Operate in the Pronunciation Task?}

Many researchers (e.g., Balota \& Lorch, 1986; de Groot, 1984, 1985; Forster, 1979, 1981; Lorch, Balota, \& Stamm, 1986; Neely \& Keefe, 1989; Seidenberg et al., 1984) assume that postlexical mechanisms have much less influence on priming in the pronunciation task than in the LDT. Perhaps the best evidence for this assumption comes from Seidenberg et al.'s (1984) finding that the pronunciation task fails to yield the backwards priming effect under conditions in which backwards priming occurs in the LDT. That is, relative to the unrelated prime pan, the prime hop facilitated processing of the target bell in the LDT. Presumably, backwards priming occurs because subjects check for an association between the target bell and the prime hop and find one via the backwards bell-hop association. Because this biases a "word" decision/ response in the backwards priming condition relative to the unrelated priming condition in which the absence of target-prime association biases a "nonword" decision/ response, priming is observed. (Backwards priming has also been obtained by Koriat, 1981, in the LDT. See also Briand, den Heyer, \& Dannenbring, 1988, and Dark, 1988 , for interesting variations on this theme.) Moreover, because knowledge that a target is unrelated to its prime does not provide useful information as to what response to make to the target in the pronunciation task, the semantic matching strategy is not used, and backwards priming does not occur. Since backwards priming is presumably produced by postlexical mechanisms, its absence in pronunciation strongly implies that postlexical priming mechanisms do not operate in that task. ${ }^{1}$

Particularly germane to the present research is Seidenberg et al.'s (1984) failure to find an RPE in a pronunciation task under conditions similar to those in which they observed an RPE in the LDT. On the basis of this finding, Seidenberg et al. concluded that the RPE in the LDT task was due to the operation of postlexical priming mechanisms rather than the prelexical expectancy mechanism. Although Seidenberg et al.'s conclusion is logically sound, the data on which it was based are open to question on both methodological and empirical grounds. As for methodology, the different RPEs obtained in their LDT and pronunciation task could have been due to procedural differences between these two tasks rather than to task differences per se. The procedural differences between tasks occurred because in creating tests for their pronunciation task, Seidenberg et al. replaced each nonword target in their LDT with a word that was unrelated to the word prime that had preceded that nonword target. As is standard in pronunciation, the nonwords were removed presumably because the inclusion of nonwords in the pronunciation task might have induced subjects (1) to emphasize nonlexical grapheme-to-phoneme cor- 
respondence rules in pronouncing the words (which might reduce priming), or (2) to make unnecessary implicit lexical decisions during pronunciation (which would possibly lead subjects to invoke postlexical priming mechanisms, thereby subverting the logic of using the pronunciation task). Because nonwords were replaced by words unrelated to their primes in the pronunciation lists, the two relatedness proportions of .50 and .29 for the LDT became .33 and .20 , respectively, for pronunciation. Perhaps, then, the failure to obtain an RPE in pronunciation was due to the .13 manipulation's being smaller than the .21 manipulation in the LDT and occurring at a lower point along the relatedness proportion continuum. Because of these methodological problems, Seidenberg et al.'s results do not unambiguously refute an expectancy interpretation of the RPE.

There are also empirical reasons for challenging Seidenberg et al.'s (1984) conclusion that the expectancy mechanism is not involved in priming. First, in their pronunciation task, Huttenlocher and Kubicek (1983) found that overall priming increased from a nonsignificant $5 \mathrm{msec}$ in an RP(.125) group to a significant $14 \mathrm{msec}$ in an RP(.875) group. (However, this was discounted because the priming $\times$ relatedness proportion interaction was not statistically significant.) Second, after the appearance of Seidenberg et al.'s (1984) results, Balota (1988), Durgunoglu (1986, Experiment 5), and Pring and Snowling (1986, Experiment 1) all reported priming in a pronunciation task, using the category-shift instructions introduced by Neely (1977), in which subjects were told to use a category-name prime (e.g., $A N I M A L$ ) as a cue to generate the expectancy that the upcoming word target would come from some prespecified unrelated category (such as color names). On most of the trials, the word target was a member of the expected category. However, on a few trials, it was a member of an unexpected category. The result was that RTs to expected targets were faster than those to unexpected targets. This effect would presumably be due to the operation of an expectancy mechanism, since postlexical processes should not be operating in pronunciation and since automatic spreading activation would not produce facilitation for a target unrelated to the prime. Particularly interesting was Durgunoglu's (1986) finding that these expectancy-based priming effects occurred for high-dominance exemplars but not for lowdominance exemplars in pronunciation. This result nicely converges with Neely et al.'s (1989) LDT finding that the effects of relatedness proportion, unconfounded with the effects of nonword ratio, are confined to priming for highdominance category exemplars.

\section{EXPERIMENT}

In the present experiment, we try to resolve the theoretical ambiguities embodied in the results just reviewed. On the one hand, we have Seidenberg et al.'s (1984) failure to find an RPE in pronunciation, which implies that expectancy does not mediate priming. On the other hand, we have (1) Huttenlocher and Kubicek's (1983) findings, which were suggestive of an RPE effect occurring in pronunciation, (2) Balota's (1988), Durgunoglu's (1986), and Pring and Snowling's (1986) expectancy-based priming effects in pronunciation, and (3) Durgunoglu's (1986) finding that expectancy-based priming in pronunciation occurs for high-but not low-dominance category exemplars. Although these results imply that expectancy can mediate priming, they do not unambiguously show that expectancy contributes to the RPE. Huttenlocher and Kubicek's (1983) results are empirically ambiguous because the priming $\times$ relatedness proportion interaction was not statistically significant. The other results are ambiguous, because they come from a situation in which subjects were explicitly instructed to use an expectancy strategy. Thus, it is still possible that expectancy plays no role in producing priming in the standard relatedness proportion paradigm, in which explicit expectancy instructions are not given. Finally, we have Neely et al.'s (1989) findings that the RPE, unconfounded with the nonword ratio effect, is confined to priming for high-dominance exemplars in the LDT. As mentioned earlier, this result is consistent with both the expectancy account and de Groot's $(1984,1985)$ postlexical account.

In the present experiment, we address these issues by examining an RPE in a pronunciation task, using exactly the same materials and procedures that Neely et al. (1989, Experiment 1) used in their LDT. To have a stronger manipulation of relatedness proportion than Seidenberg et al. (1984), we tested RP(.33) and RP(.88) groups. To determine if the presence of nonwords in a pronunciation task influences the RPE (by perhaps inducing subjects to make unnecessary implicit lexical decisions), we also tested $\mathrm{RP}(.33)$ and $\mathrm{RP}(.88)$ groups with all of the nonwords removed from the list. Unlike Seidenberg et al., we did not replace the nonwords with words unrelated to their primes, for this would have changed the relatedness proportion manipulation. Although relatedness proportion and the nonword ratio were confounded in the two groups that received nonwords, this should not be important, because the semantic matching mechanism, which is influenced by the nonword ratio, should not be operating in pronunciation. (As will be described shortly, we have internal checks for this claim.)

If Seidenberg et al.'s (1984) claim that expectancy does not contribute to the RPE in the standard LDT and pronunciation task (in which explicit expectancy instructions are not given) is true, an RPE should be obtained for neither high-nor low-dominance exemplars in our pronunciation task, particularly when nonword targets are excluded. If an RPE occurs when nonword targets are included, it could be argued that the inclusion of nonword targets turns the pronunciation task into an implicit LDT, thereby invoking the postlexical mechanisms that produce the RPE in the LDT.

On the other hand, if Neely et al.'s (1989) expectancy analysis is correct, an RPE should be observed in the present pronunciation tasks, regardless of whether non- 
word targets are or are not excluded. More importantly, since in the present experiment we used exactly the same materials that Neely et al. (1989) used in their LDT, we can make exact predictions concerning the size of the RPE we should observe. Because we assume that the postlexical semantic matching mechanism, which is influenced by the nonword ratio, does not operate in pronunciation (even when nonwords are present), our predictions about the sizes of the RPEs that should occur for the high- and lowdominance exemplars are based only on changes in the relatedness proportion and not on changes in the nonword ratio. (Thus, as noted earlier, these predictions provide a test of the assumption that the nonword ratio, which is confounded with the relatedness proportion in the groups receiving nonword targets, has no effect on the results.) If expectancy plays a role in producing the RPE, and if expectancy operates similarly in the LDT and in pronunciation (as Neely and Keefe, 1989, have argued), Neely et al.'s (1989) regression analyses make the following predictions for the present pronunciation task: For highdominance exemplars, the .55 increase in relatedness proportion (from .33 to .88 ) should result in a $22-\mathrm{msec}$ RPE (because every .10 increment in relatedness proportion produces a 4-msec increase in overall priming, due to expectancy). For low-dominance exemplars, which are not included in the expectancy set, and for which relatedness proportion had no effect on priming in Neely et al.'s (1989) LDT, the RPE should be $0 \mathrm{msec}$. (The 29-msec RPE that occurred for the low-dominance exemplars in Neely et al.'s LDT was presumably due to the confounded effects of the nonword ratio, which should have no effect in the present pronunciation task, because the semantic matching mechanism does not operate in this task.)

In summary, in the present experiment, we tested contrasting predictions derived from opposing views concerning whether or not the expectancy mechanism plays a role in the RPE. The view that expectancy does not play a role predicts a 0-msec RPE for both high- and low-dominance exemplars when nonword targets are excluded from our pronunciation task. (When nonwords are included, this view allows for RPEs of unspecified magnitudes occurring for the high- and low-dominance exemplars.) On the other hand, Neely et al.'s (1989) view that expectancy plays a similar role in producing RPEs in the LDT and pronunciation predicts a 22-msec RPE for high-dominance exemplars and a 0-msec RPE for low-dominance exemplars, whether or not nonwords are excluded from the pronunciation task.

\footnotetext{
Method

Design. For word targets, the design was a 2 (relatedness proportion: .33 vs. .88 ) $\times 2$ (nonwords present vs. absent) $\times 3$ (prime type: related, unrelated, neutral) $\times 2$ (target-category dominance: high vs. low) design, with the first two factors manipulated between subjects and the latter two manipulated within subjects. For groups receiving nonword targets, the design for nonword targets was a 2 (relatedness proportion: .33 vs. .88 ) $\times 2$ (prime type: word prime
}

vs. neutral prime condition), with the first factor manipulated between subjects and the second factor manipulated within subjects.

Stimulus materials and list construction. The lists used in this experiment were exactly those Neely et al. (1989, Experiment 1) used in their $\mathrm{RP}(.33)$ and $\mathrm{RP}(.88)$ groups. The category-name prime and category-exemplar target words came from the Battig and Montague (1969) and Shapiro and Palermo (1970) norms. Four highdominance and 4 low-dominance exemplars were selected from each of 48 categories to obtain an adequate number of observations in the priming conditions with a low probability. Because many of these categories were relatively small, it was not possible to equate the high- and low-dominance targets for word frequency. The Kucera-Francis (1967) frequency counts for the high- and lowdominance targets were 40.5 and 14.9 , respectively. (The implications of this confounding between dominance and frequency will be discussed when the results are presented.)

For the two groups that received nonword targets, the 768-trial base list consisted of 384 nonrepeated word targets and 384 nonrepeated nonword targets. Pronounceable nonword targets were constructed by randomly changing one or two letters in category exemplars other than those selected as word targets. A nonword target never followed a prime that was the name of the category of which the word used to create that nonword was a member. The same targets, presented in the same order, were used in both relatedness proportion groups. The only thing that differed across groups was that the primes were rotated for word targets to yield the two different relatedness proportions. For each dominance level of the word targets (high vs. low) in the RP(.33) versus RP(.88) groups, respectively, there were 48 versus 112 related priming trials (in which the target was preceded by the name of the category of which it was a member), 96 versus 16 unrelated priming trials (in which the target was preceded by the name of a category of which that target was not a member), and 48 versus 64 neutral priming trials (in which the prime was XXX). For nonword targets, there were 288 versus 256 category-name priming trials and 96 versus 128 neutral-prime trials in the $\operatorname{RP}(.33)$ and $\mathrm{RP}(.88)$ groups, respectively. Although there were more category-name primes than neutral primes, word and nonword targets were equally likely following each of these two kinds of primes.

For counterbalancing purposes, a subset of the word and nonword target trials was designated as critical. In the RP(.33) group, there were 48 critical word targets at each dominance level in each of the three priming conditions. However, because there could be only 16 total word targets at each dominance level in the unrelated priming condition in the RP(.88) group, 16 of the 48 targets used as critical targets in the RP(.33) group were selected as critical targets in the RP(.88) group. To counterbalance these critical target items across the different priming conditions within each of the $\mathrm{RP}(.33)$ and $\mathrm{RP}(.88)$ groups, five additional list versions were constructed from each of the two base lists described above, by exchanging primes for critical targets across lists. For the critical trials, prime type was alternated across the six list versions such that each word target appeared twice each in the related, unrelated, and neutral priming conditions, and each nonword target appeared three times in the word-prime and neutral-prime conditions. Each category name served as a related and unrelated prime and was used as a prime an equal number of times preceding a word or nonword target. The unrelated primes were selected so that they did not offer information about the category membership of the target that would follow (e.g., the category-name FRUIT did not consistently serve as an unrelated prime for exemplars of the "sports" category.) Only the data from the critical trials were included in the statistical analyses.

For each proportion group, the testing session began with the same practice block of 24 trials, with 12 word targets and 12 nonword targets. Four of the word targets were preceded by a related 
prime, 4 by an unrelated prime, and 4 by a neutral prime, with 2 targets within each priming condition being high-dominance exemplars and 2 being low-dominance exemplars. Six of the nonword targets were preceded by a word prime and 6 by a neutral prime. In this practice list, all word targets used were taken from four categories not used in the test lists, with the category names of these targets being used as related and unrelated primes.

The two groups not receiving nonword targets were given exactly the same lists as those just described, except that the primes and targets for all nonword target trials were removed from the test list.

Procedure. Procedures were identical to those used by Neely et al. (1989). Subjects were read general instructions describing the task and were told that the word targets were common English words. They were also told to pay close attention to the primes, although they were not to respond to them. Depending on the relatedness proportion, the subjects were told that either most or some of the word targets would be related to the prime, and that they should use this information to optimize their performance. They were also given an example of a related category prime and exemplar target not used in the test lists. List presentation was controlled by a Cromemco Z-2D microcomputer equipped with a FLASHWRITER video board with a hardware modification that synchronized stimulus presentation with the reaction time clock. The subjects wore headsets equipped with Telex CS-91 microphones and connected to a Lafayette Voice Activated Relay, Model 18010. Primes were presented in uppercase for $500 \mathrm{msec}$ in the middle of the screen. After the prime disappeared, the screen was blank for $500 \mathrm{msec}$, and then the target appeared in lowercase in the same location on the screen for 1,000 msec. The screen was then blank for $3,000 \mathrm{msec}$, and the next trial began. If no response was given to the target within 4 sec of the target onset, the computer recorded that no response occurred. The subjects were asked to pronounce the target aloud in a phonetically acceptable manner. The experiment lasted about $85 \mathrm{~min}$. A break of about $1 \mathrm{~min}$ was given after the practice list and after each sixth of the longer test list.

Subjects. Seventy-two Purdue students served in each of the two groups receiving nonword targets, and 36 served in each of the two groups not receiving nonword targets. Approximately half of the students in each group were paid $\$ 7.50$ for their participation (some of these were graduate students), while the other half participated in partial fulfillment of an introductory psychology course requirement for research participation or library research.

\section{Results and Discussion}

Preliminary analyses: Facilitation and inhibition effects. RTs for incorrect responses and RTs that fell beyond two standard deviations from a subject's mean RT for a "word" or "nonword" response were excluded from the analyses. ${ }^{2}$ Because the primary data of interest are the priming effects for RTs for word targets, and because the error rates for word targets were very low (ranging from $0.1 \%$ to $2.0 \%$ ) and varied inconsistently with our experimental manipulations, Table 1 presents the priming effects only for RTs for word targets in the related and unrelated priming conditions relative to the neutral priming condition, as well as the overall priming effect. (Those interested in the general speed of responding may refer ahead to Table 2, which gives the RTs for word targets in the neutral priming condition and the RTs for nonword targets that followed neutral primes and word primes.)

To evaluate the statistical significance of a particular facilitation or inhibition effect in a related or unrelated priming condition relative to the neutral priming condition, four separate 3 (priming condition) $\times 2$ (target dominance) analyses of variance (ANOVAs) were performed on the data from each of the four groups. Because the statistical significance of each priming effect was determined by a least significant difference $(L S D)$ based on the $M S_{\mathrm{e}}$ for the priming condition $\times$ target dominance interaction from these separate ANOVAs, slightly different magnitudes of priming were required in order to reach

Table 1

Mean Priming Effects

for Reaction Times (in milliseconds) in the Related and Unrelated Priming Conditions Relative to the Neutral Priming Condition and Mean Overall Priming Effects

\begin{tabular}{|c|c|c|c|c|c|c|}
\hline \multirow{3}{*}{$\begin{array}{l}\text { Nonword Condition } \\
\text { and RP Group }\end{array}$} & \multicolumn{6}{|c|}{ Type of Target and Type of Priming } \\
\hline & \multicolumn{3}{|c|}{ High-Dominance Word Targets } & \multicolumn{3}{|c|}{ Low-Dominance Word Targets } \\
\hline & Related & Unrelated & Overall & Related & Unrelated & Overall \\
\hline \multicolumn{7}{|c|}{ Nonwords Absent } \\
\hline $\begin{array}{l}\mathrm{RP}(.88) \\
\mathrm{RP}(.33) \\
\mathrm{RPE}\end{array}$ & $\begin{array}{l}+30^{*} \\
+19^{*}\end{array}$ & $\begin{array}{l}-2 \\
+5\end{array}$ & $\begin{array}{l}+32^{*} \\
+14^{*} \\
+18^{*}\end{array}$ & $\begin{array}{c}+6 \\
+18^{*}\end{array}$ & $\begin{array}{c}-4 \\
+10^{*}\end{array}$ & $\begin{array}{l}+10 \\
+8 * \\
+2\end{array}$ \\
\hline \multicolumn{7}{|c|}{ Nonwords Present } \\
\hline $\begin{array}{l}\mathrm{RP}(.88) \\
\mathrm{RP}(.33) \\
\mathrm{RPE}\end{array}$ & $\begin{array}{l}+35^{*} \\
+26^{*}\end{array}$ & $\begin{array}{c}+3 \\
+13^{*}\end{array}$ & $\begin{array}{l}+32^{*} \\
+13^{*} \\
+19^{*}\end{array}$ & $\begin{array}{l}+18^{*} \\
+15^{*}\end{array}$ & $\begin{array}{l}+12^{*} \\
+10^{*}\end{array}$ & $\begin{array}{l}+6 \\
+5^{*} \\
+1\end{array}$ \\
\hline
\end{tabular}

Note-The priming effects for the related and unrelated priming conditions were computed by subtracting the mean reaction times (RTs) in these two conditions from the mean RT in the neutral priming condition. Thus, a positive value indicates facilitation and a negative value, inhibition. The overall priming effect is the RT in the unrelated condition minus the RT in the related condition. Thus, it is the related priming effect minus the signed unrelated priming effect, with a positive score indicating facilitation. An * indicates a priming effect was significant at the $p<.05$ level. Because the statistical significance of the priming effects was evaluated separately for the four different groups (see text), priming effects with similar magnitudes were sometimes significant, and sometimes not. RPE = relatedness proportion effect, which was computed by subtracting overall priming for the $\operatorname{RP}(.33)$ group from that for the $\operatorname{RP}(.88)$ group. 
statistical significance in the four different groups. (In all statistical analyses, we adopted the $p<.05$ level of significance.)

As can be seen in Table 1, significant facilitation effects occurred in all related priming conditions except for the low-dominance targets in the RP( $\left.{ }^{88}\right)$ group in which nonwords were not presented. However, some of these facilitation effects should be discounted, because significant facilitation sometimes occurred in the corresponding unrelated priming condition. Because of this, we turn to the more informative overall priming effects to determine whether or not our pronunciation task yielded an RPE. (We defer to the Discussion section a consideration of the implications of our failing to find significant inhibition effects in our unrelated priming conditions.)

Overall priming effects and the RPE. As shown in Table 1, the overall priming effects confirm the prediction derived from Neely et al.'s (1989) expectancy analysis that an RPE will occur for high- but not lowdominance exemplars, regardless of whether or not nonword targets are excluded. Specifically, regardless of whether or not nonword targets were excluded from the test list, overall priming was 18 to $19 \mathrm{msec}$ greater in the $\mathrm{RP}(.88)$ groups than in the RP(.33) groups (a significant RPE) for high-dominance targets, whereas overall priming was only 1 to $2 \mathrm{msec}$ greater (a nonsignificant RPE) for low-dominance targets. Moreover, the 18- to 19-msec RPEs for high-dominance targets and the 1- to 2-msec RPEs for low-dominance targets are remarkably close to the 22- and 0-msec RPEs predicted on the basis of the regression equations that Neely et al. obtained in their LDT for "pure" RPEs, uncontaminated by nonword ratio effects. To provide statistical support for the above conclusions, we excluded data from the neutral priming conditions and performed a 2 (relatedness proportion: .88 vs. .33) $\times 2$ (nonwords: present vs. absent) $\times 2$ (priming: related vs. unrelated conditions) $\times 2$ (dominance: high- vs. low-dominance targets) unequal $N$ s mixed-factor ANOVA on the RT data. In support of our conclusions, this ANOVA revealed that the relatedness proportion $x$ priming $\times$ dominance interaction was indeed significant $\left[F(1,212)=8.36, M S_{\mathrm{e}}=427.8\right]$, whereas the relatedness proportion $\times$ priming $\times$ dominance $\times$ nonwords interaction did not approach significance $(F<1)$. Clearly, then, these data provide rather striking support for Neely and Keefe's (1989) and Neely et al.'s (1989) view that priming is mediated by an expectancy mechanism, a point on which we elaborate in the Discussion section.

A 2 (relatedness proportion: .88 vs. .33 ) $\times 2$ (nonwords: present vs. absent) $\times 2$ (dominance: high- vs. lowdominance targets) unequal $N \mathrm{~s}$ mixed-factor ANOVA was performed on RTs for the word targets in the neutral priming conditions (see Table 2). This analysis allows us to determine if the differences in the overall priming effects that occurred as a function of relatedness proportion and target dominance were associated with differences in overall RTs. It is important to address this question, because priming effects typically increase as overall RTs increase (e.g., see Durgunoglu, 1988, Hines, Czerwinski, Sawyer, \& Dwyer, 1986, Shulman \& Davison, 1977; Stanovich \& West, 1979, 1983). This analysis showed that although RTs were somewhat faster when nonwords were absent versus present (655 vs. $671 \mathrm{msec}$ ), this effect was not statistically significant $\left[F(1,212)=1.44, M S_{c}=17,986.3\right]$. The only effect that was significant was the effect of dominance $\left[F(1,212)=137.45, M S_{\mathrm{e}}=438.6\right]$, with RTs being $26 \mathrm{msec}$ faster to high- than to low-dominance targets. Most or all of this dominance effect is likely due to the high-dominance targets' being higher frequency words than the low-dominance targets were (see Method section). However, because high-frequency words typically yield smaller priming effects than low-frequency words do (e.g., see Becker, 1979; Stanovich \& West, 1981, 1983), our finding that priming effects were larger for high- than for low-dominance targets cannot be attributed to dominance's having been confounded with word frequency. Also, the RPEs that occurred for highdominance targets cannot be due to a speed-of-processing artifact, since RTs to high-dominance targets were slightly slower in the RP(.33) groups than in the RP(.88) groups.

Table 2

Mean Reaction Times (in milliseconds) for Word Targets in the Neutral-Prime Condition and for Nonword Targets in the Neutral-Prime and Word-Prime Conditions and Nonword Facilitation Effects (NWFEs)

\begin{tabular}{ccccccc}
\hline & \multicolumn{5}{c}{ Type of Prime and Type of Target } \\
\cline { 2 - 5 } $\begin{array}{c}\text { Nonword Condition } \\
\text { and RP Group }\end{array}$ & $\begin{array}{c}\text { XXX- } \\
\text { HD Word }\end{array}$ & $\begin{array}{c}\text { XXX- } \\
\text { LD Word }\end{array}$ & $\begin{array}{c}\text { XXX- } \\
\text { Nonword }\end{array}$ & $\begin{array}{c}\text { Word } \\
\text { Prime-Nonword }\end{array}$ & NWFE \\
\hline \multicolumn{7}{c}{$\begin{array}{c}\text { Nonwords Absent } \\
\text { RP(.88) }\end{array}$} \\
\hline RP(.33) & 641 & 668 & - & - & - \\
& 640 & 672 & - & - & - \\
RP(.88) & 653 & 677 & 788 & 806 & $+18^{*}$ \\
RP(.33) & 666 & 688 & 783 & 800 & $+17^{*}$ \\
\hline
\end{tabular}

Note-XXX-HD Word $=$ neutral prime, high-dominance word target; XXX-LD Word $=$ neutral prime, low-dominance word target; XXX-Nonword = neutral prime, nonword target; Word Prime-Nonword $=$ word prime, nonword target. An * indicates the NWFE was significant at the $p<.05$ level. 
Nonword data. Error rates for nonword targets were higher than they were for word targets in the groups that received nonword targets. Specifically, in the RP(.88) group, the error rates were $7.1 \%$ and $7.7 \%$ in the wordprime and neutral-prime conditions, respectively; in the $\mathrm{RP}(.33)$ group, the corresponding error rates were $4.5 \%$ and $4.2 \%$. As indicated by a 2 (relatedness proportion: .88 vs. .33 ) $\times 2$ (type of prime: word vs. neutral prime) mixed-factor ANOVA on the RTs, the only significant effect was that RTs were $18 \mathrm{msec}$ faster when the nonword targets followed a word prime rather than a neutral prime $\left[F(1,142)=37.46, M S_{\mathrm{e}}=598.9\right]$. However, it is important that the nonword facilitation effects were virtually identical in the $\operatorname{RP}(.33)$ and $\operatorname{RP}(.88)$ groups (i.e., $17 \mathrm{vs} .18 \mathrm{msec}$ ). We will consider the implications of a nonword facilitation effect for pronunciation in the Discussion section.

\section{DISCUSSION}

The empirical import of the present results is that they clearly demonstrate an RPE for high-dominance exemplars in a pronunciation task, whether or not it includes nonword targets. Our finding that priming for highdominance exemplars increased by $19 \mathrm{msec}$ as relatedness proportion increased from .33 to .88 corroborates Huttenlocher and Kubicek's (1983) finding that priming increased from a nonsignificant $5 \mathrm{msec}$ to a significant $14 \mathrm{msec}$ as relatedness proportion increased from .13 to .88 in their pronunciation task. However, our RPE for high-dominance exemplars stands in contrast to Seidenberg et al.'s (1984) failure to find an RPE in pronunciation. Their failure to find an RPE was likely due to their relatedness proportion manipulation of .13 (from .20 to .33 ) being much weaker than the one used here and in Huttenlocher and Kubicek (1983). It cannot be due to their not having included nonword targets, since we obtained an RPE when nonword targets were excluded from our test lists. Nor is it likely due to their having used different materials, which were strongly associatively related, as were our category-name primes and high-dominance exemplars, for which we obtained the RPE.

From a theoretical perspective, the most interesting aspect of the present results is that an RPE occurred for high-dominance targets but not for low-dominance targets. As outlined in the introduction, this result strongly supports Neely and Keefe's (1989) view that a "pure" RPE, uncontaminated by the confounded effects of the nonword ratio, is mediated by an expectancy mechanism that contributes to priming in both the LDT and the pronunciation task. If the "pure" RPE in the LDT were due solely to postlexical processes, it should not have occurred in the pronunciation task, which presumably minimizes the operation of such processes. Moreover, the present data provide a rather striking cross-validation of the slopes of the "pure" relatedness proportion effects, uncontaminated by nonword ratio effects, that Neely et al. (1989) obtained in regression analyses of their LDT data.
Specifically, if the postlexical semantic matching strategy, which is modulated by the nonword ratio, plays no role in producing priming in pronunciation, any RPEs observed in pronunciation must be due solely to expectancy, which is modulated by "pure" relatedness proportion. On the basis of Neely et al.'s (1989) slopes for "pure" RPEs, one can predict a 22-msec RPE for high-dominance exemplars and a 0-msec RPE for low-dominance exemplars in the current experiment. These values are remarkably close to the 18 - to $19 \mathrm{msec}$ and 1- to 2-msec RPEs that we observed. That Neely et al. (Experiment 1) observed a 29-msec RPE for their low-dominance exemplars in their LDT is presumably due to the operation of the postlexical semantic matching mechanism in that task being influenced by the nonword ratio, which was confounded with relatedness proportion.

Because Neely et al. (1989) and Neely and Keefe (1989) have argued that the nonword facilitation effect in the LDT is produced by the semantic matching strategy, the nonword facilitation effect that we observed here is potentially problematic for our claim that the semantic matching strategy was not operating in our pronunciation task. However, if the semantic matching strategy were producing the present nonword facilitation effects, we might have expected to have observed more nonword facilitation in the RP(.88) group than in the RP(.33) group, because of the higher nonword ratio in the former group (.89 vs. .60). Indeed, with these same groups and materials, Neely et al. observed $13 \mathrm{msec}$ more nonword facilitation in the $\mathbf{R P}(.88)$ group than in the $\mathrm{RP}(.33)$ group (though this effect was not significant). By comparison, the nonword facilitation effect was only $1 \mathrm{msec}$ larger in the $\operatorname{RP}(.88)$ group than in the RP(.33) group in the present data. Thus, it may be that our nonword facilitation effects were due to our neutral condition's being somehow inappropriate (e.g., see Jonides \& Mack, 1984). Perhaps the activation of articulatory programs by word primes leads to a generalized priming of the articulators, which speeds up pronunciation latencies to subsequent targets. This would also account for the anomalous facilitation effects we obtained in some of our unrelated priming conditions, a result to which we now turn.

Our failure to find inhibition effects in our unrelated priming conditions would seem to undermine our claim that an expectancy mechanism produced our RPEs, at least if one accepts Posner and Snyder's (1975) analysis that inhibition effects for unexpected (unrelated) targets are necessary for inferring that expectancies are contributing to priming. In this regard, it is interesting that with the same materials used here, Neely et al. (1989) obtained a 16-msec inhibition effect for high-dominance exemplars ( $p<.05$, one-tailed) and a 21-msec inhibition effect for low-dominance exemplars $(p<.05$, two-tailed $)$ in the unrelated priming condition in their $\mathrm{RP}(.88) / \mathrm{NR}(.89)$ group, in which expectancy should have been operating due to the high relatedness proportion. Although substantial inhibition typically occurs in unrelated priming conditions in the LDT when category names are used as 
primes and both high-and low-dominance exemplars are used as related targets, inhibition does not occur in lists in which related primes and targets are always strongly associatively related (e.g., see Becker, 1980, den Heyer, Briand, \& Smith, 1985, Smith, Briand, Klein, \& den Heyer, 1987). Becker's (1980) expectancy theory explains inhibition as being due to subjects' wasting their time unsuccessfully searching the expectancy set following unrelated primes, relative to the neutral priming condition in which there is no expectancy set to waste time searching through. In his analysis, inhibition effects are larger for category materials than for strong associates, because the former materials induce subjects to generate larger expectancy sets.

An alternative to Becker's (1980) approach is to assume that expectancy sets are small even with category materials (i.e., with only a few high-dominance exemplars being included in the expectancy set) and that the inhibition that occurs with these materials in the LDT is due to the semantic matching mechanism. One would then need to assume that semantic matching is more likely when the semantic matching mechanism can often find a backward association to the prime from a target not included in the expectancy set, as would be so when low-dominance exemplars are targets. If semantic matching does indeed not occur in pronunciation, this analysis would also explain why inhibition effects typically do not occur in pronunciation (cf. Forster, 1981; Lorch et al., 1986; West \& Stanovich, 1982; the present results). ${ }^{3}$

Clearly, our claim that expectancy contributes to the RPE in the pronunciation task is contrary to conclusions Seidenberg et al. (1984) reached on the basis of the data they had available to them at that time. However, we believe that more recent results (e.g., Balota, 1988; Durgunoglu, 1986; Neely et al., 1989; Pring \& Snowling, 1986; the present data) strongly indicate that in order to account for priming effects in pronunciation, one cannot ignore that subjects will use a prime to generate an expectancy set containing items related to that prime when it is advantageous for them to do so.

\section{REFERENCES}

BALOTA, D. A. (1988). The temporal influence of semantic relatedness, expectancy, and age in a semantic priming pronunciation task. Unpublished manuscript.

BALOtA, D. A., \& LoRCh, R. F., JR. (1986). Depth of automatic spreading activation: Mediated priming effects in pronunciation but not in lexical decision. Journal of Experimental Psychology: Learning, Memory, \& Cognition, 12, 336-345.

Battig, W. F., \& Montague, W. E. (1969). Category norms for verbal items in 56 categories: A replication and extension of the Connecticut category norms. Journal of Experimental Psychology Monographs, 80(3, Pt. 2).

BECKer, C. A. (1979). Semantic context and word frequency effects in visual word recognition. Joumal of Experimental Psychology: Human Perception \& Performance, 5, 252-259.

BECKER, C. A. (1980). Semantic context effects in visual word recognition: An analysis of semantic strategies. Memory \& Cognition, $\mathbf{8}$, 493-512

Briand, K., den Heyer, K., \& Dannenbring, G. L. (1988). Retro- active semantic priming in a lexical decision task. Quarterly Journal of Experimental Psychology, 40A, 341-359.

DARK, V. J. (1988). Semantic priming, prime reportability, and retroactive priming are interdependent. Memory \& Cognition, 16, 299-308.

DE Groot, A. M. B. (1984). Primed lexical decision: Combined effects of the proportion of related prime-target pairs and the stimulusonset asynchrony of prime and target. Quarterly Journal of Experimental Psychology, 36A, 253-280.

DE GROOT, A. M. B. (1985). Word-context effects in word naming and lexical decision. Quarterly Joumal of Experimental Psychology, 37A, 281-297.

de Groot, A. M. B., Thomassen, A. J. W. M., \&udson, P. T. W. (1982). Associative facilitation of word recognition as measured from a neutral prime. Memory \& Cognition, 10, 358-370

DEN HEYER, K. (1985). On the nature of the proportion effect in semantic priming. Acta Psychologica, 60, 25-38.

den Heyer, K., Briand, K., Dannenbring, G. L. (1983). Strategic factors in a lexical-decision task: Evidence for automatic and attention-driven processes. Memory \& Cognition, 11, 374-381.

DEN HeYer, K., Briand, K. , SMith, L. (1985). Automatic and strategic effects in semantic priming: An examination of Becker's verification model. Memory \& Cognition, 13, 228-232.

DuRgunoglu, A. Y. (1986). A comparison of the two versions of the interactive-compensatory model of reading. Unpublished doctoral dissertation, Purdue University.

DURGUNOGLU, A. Y. (1988). Repetition, semantic priming, and stimulus quality: Implications for the interactive-compensatory reading model. Journal of Experimental Psychology: Learning, Memory, \& Cognition, 14, 590-603

FoRster, K. I. (1979). Levels of processing and the structure of the language processor. In W. E. Cooper \& E. C. T. Walker (Eds.), Sentence processing: Psycholinguistic studies presented to Merrill Garrett (pp. 27-85). Cambridge, MA: MIT Press

ForSTER, K. I. (1981). Priming and the effects of sentence and lexical contexts on naming time: Evidence for autonomous lexical processing. Quarterly Journal of Experimental Psychology, 33A, 465-495.

Hines, D., Czerwinski, M., SAWyer, P. K., \& DWyer, M. (1986). Automatic semantic priming: Effect of category exemplar level and word association level. Joumal of Experimental Psychology: Human Perception \& Performance, 12, 370-379.

HUTTENLOCHER, J., KUBiCEK, L. (1983). The source of relatedness effects on naming latency. Journal of Experimental Psychology: Leaming, Memory, \& Cognition, 9, 486-496

JoNIDES, J., \& MACK, R. (1984). On the cost and benefit of cost and benefit. Psychological Bulletin, 96, 29-44.

KorIAT, A. (1981). Semantic facilitation in lexical decision as a function of prime-target association. Memory \& Cognition, 9, 587-598.

KuČera, H., \& Francis, W. N. (1967). Computational analysis of present day American English. Providence, RI: Brown University Press.

Lorch, R. F., Jr., Balota, D. A., \& Stamm, E. G. (1986). Locus of inhibition effects in the priming of lexical decisions: Pre- or postlexical access? Memory \& Cognition, 14, 95-103.

Meyer, D. E., \& SchvaneveldT, R. W. (1971). Facilitation in recognizing pairs of words: Evidence of a dependence between retrieval operations. Journal of Experimental Psychology, 90, 227-234.

NeELy, J. H. (1976). Semantic priming and retrieval from lexical memory: Evidence for facilitatory and inhibitory processes. Memory \& Cognition, 4, 648-654.

Neely, J. H. (1977). Semantic priming and retrieval from lexical memory: Roles of inhibitionless spreading activation and limited-capacity attention. Journal of Experimental Psychology: General, 106, 226-254.

NeELY, J. H. (in press). Semantic priming effects in visual word recognition: A selective review of current findings and theories. In D. Besner \& G. Humphreys (Eds.), Basic processes in reading: Visual word recognition. Hillsdale, NJ: Erlbaum.

NeELy, J. H., \& KeEFE, D. E. (1989). Semantic context effects on visual word processing: A hybrid prospective/retrospective processing theory. In G. H. Bower (Ed.), The psychology of learning and motivation: Advances in research and theory (Vol. 24, pp. 207-248). New York: Academic Press 
Neely, J. H., Keefe, D. E., Ross, K. (1989). Semantic priming in the lexical decision task: Roles of prospective prime-generated expectancies and retrospective semantic matching. Journal of Experimental Psychology: Learning, Memory, \& Cognition, 15, 1003-1019.

NorRIS, D. (1986). Word recognition: Context effects without priming. Cognition, 22, 93-136.

Peterson, R. R., Simpson, G. B. (1989). The effect of backward priming on word recognition in single-word and sentence contexts. Journal of Experimental Psychology: Learning, Memory, \& Cognition, 15, 1020-1032.

PosNer, M. I., \& SNYDER, C. R. R. (1975). Attention and cognitive control. In R. L. Solso (Ed.), Information processing and cognition: The Loyola Symposium (pp. 55-85). Hillsdale, NJ: Erlbaum.

Pring, L., \& SNowling, M. (1986). Developmental changes in word recognition: An information processing account. Quarterly Journal of Experimental Psychology, 38A, 395-418.

RATCLIFF, R., MCKoON, G. (1988). A retrieval theory of priming in memory. Psychological Review, 95, 385-408.

Seidenderg, M. S., Waters, G. S., Sanders, M., Langer, P. (1984). Pre- and postlexical loci of contextual effects on word recognition. Memory \& Cognition, 12, 315-328.

Shapiro, S. I., \& Palermo, D. S. (1970). Conceptual organization and class membership: Normative data for representatives of 100 categories. Psychonomic Monograph Supplements, 3(11, Whole No. 43), 107-127.

Shulman, H. G., d Davison, T. C. B. (1977). Control properties of semantic coding in a lexical decision task. Journal of Verbal Learning \& Verbal Behavior, 16, 92-98.

Smith, L. C., Briand, K., Klein, R. M., \& Den Heyer, K. (1987). On the generality of Becker's verification model. Canadian Joumal of Psychology, 41, 379-386.

Stanovich, K. E., \& WeST, R. F. (1979). Mechanisms of sentence context effects in reading: Automatic activation and conscious attention. Memory \& Cognition, 7, 77-85.

Stanovich, K. E., WESt, R. F. (1981). The effect of sentence context on ongoing word recognition: Tests of a two-process theory. Journal of Experimental Psychology: Human Perception \& Performance, 7, 658-672.

STANovich, K. E., \& WeST, R. F. (1983). On priming by a sentence context. Journal of Experimental Psychology: General, 112, 1-36.
Tweedy, J. R., Lapinski, R. H., \& SchVaneveldt, R. W. (1977). Semantic-context effects on word recognition: Influence of varying the proportion of items presented in an appropriate context. Memory \& Cognition, 5, 84-89.

WEST, R. F., \& STANOVICH, K. F. (1982). Source of inhibition in experiments on the effect of sentence context on word recognition. Joumal of Experimental Psychology: Learning, Memory, \& Cognition, 8, 385-399.

\section{NOTES}

1. Recently, Peterson and Simpson (1989) obtained backwards priming in a pronunciation task under rather circumscribed conditions-that is, at a very short SOA with an auditory single-word prime (but not when that same word prime appeared as the last word in a sentence). Although it is unclear to us what mechanism(s) mediated the backwards priming effect that Peterson and Simpson obtained in their pronunciation task, in our opinion there is at present no compelling evidence to support the claim that the semantic matching mechanism contributes to priming in the pronunciation task, particularly when visual primes are used at the long SOAs that yield the RPE and the backward priming effect in the LDT.

2. The mean RTs reported here are slightly different from those reported in our convention presentations, because those means included extreme scores whereas the present means do not. However, the pattern of priming effects is the same for both of these measures.

3. That inhibition effects are typically absent in pronunciation does not mean that expectancies can never produce inhibition in that task. For example, Pring and Snowling (1986) obtained significant expectancybased inhibition effects in pronunciation when children were given ex plicit expectancy instructions. Thus, the delineation of the exact conditions under which expectancies (uncontaminated by the operation of the postlexical semantic matching mechanism) will produce inhibition merits the expenditure of additional research efforts.

(Manuscript received August 19, 1988; revision accepted for publication September 11, 1989.) 